Paediatr Paedolog 2016 51:156-161

DOI 10.1007/s00608-016-0396-2

Online publiziert: 29. August 2016

(c) Der/die Autor(en) 2016. Dieser Artikel ist eine Open-Access-Publikation.

CrossMark
Marie Peterseil · Wolfgang Gunzer - Bianca Fuchs-Neuhold

Health Perception Lab, Institut Diätologie, FH Joanneum, Graz, Österreich

\title{
Einflussfaktoren auf die Geschmacksentwicklung von Säuglingen
}

\begin{abstract}
Ernährungsbedingte Erkrankungen, wie die Adipositas, werden im 21. Jahrhundert als Hauptgesundheitsproblem angesehen. Für die Entwicklung von Herz-Kreislauf-Erkrankungen, Schlaganfall, Diabetes mellitus sowie anderen nichtübertragbaren Krankheiten stellen Übergewicht und Adipositas einen hohen Risikofaktor dar. In der europäischen Union sind laut aktuellen Zahlen der Weltgesundheitsorganisation (WHO) $50 \%$ aller Europäer übergewichtig oder adipös.
\end{abstract}

In Österreich sind mehr als jeder zweite Mann (52,3\%) und beinahe ein Drittel aller österreichischen Frauen (27,6 \%) übergewichtig oder adipös. Fast ein Viertel aller 7- bis 14-jährigen Schulkinder in Österreich ist übergewichtig oder adipös. Weltweit ist die Prävalenz von Übergewicht und Adipositas bei Kindern unter fünf Jahren am Zunehmen. Diese alarmierenden Zahlen spiegeln sich auch in den immensen finanziellen Belastungen, die dadurch für das Gesundheitssystem entstehen, wider: Im Jahr 2004 ergaben sich in Österreich basierend auf internationalen Kostenschätzungen adipositasassoziierte Gesundheitsausgaben von 227,7 Mio. bis 1,1 Mrd. Euro. Diese Entwicklung erfordert effektive Präventionsstrategien. Im aktuellen Global Status Report on Noncommunicable Diseases der

Substantial contributions (inalphabeticalorder): Buchinger, E., Grach, D., Konrad, M., Kronberger, A., Lederer, E., Martin, J., Maunz, S., Pail, E., Rath, A., Riederer, M., Schweighofer, N., van der Kleyn, M., Waldner, I., Wallner, M.

Ausführliche Literatur sowie Text inkl. Verweisen bei den Verfassern
WHO wurde nochmals mit Nachdruck darauf hingewiesen, effektive Strategien zur Bekämpfung der steigenden Adipositasprävalenz einzusetzen und vor allem der rapiden Zunahme von Adipositas unter Kindern entgegenzuwirken.

\section{"Metabolische Program- mierung"}

Bereits während der pränatalen und frühen postnatalen Entwicklung haben Faktoren wie maternale Ernährung, Hormone usw. einen prägenden Einfluss auf die späteren Stoffwechsel- und Organfunktionen. In diesem Zusammenhang wird auch von „metabolischer Programmierung“ gesprochen. Ein noch junges Forschungsfeld, das den Zusammenhang zwischen Adipositas, möglichen prägenden Einflüssen aus Nahrungsmittelauswahl und eventuell veränderten Geschmackspräferenzen untersucht, ist das der gesundheitsorientierten Lebensmittelsensorik. Wissenschaftliche Untersuchungen in diesem Bereich beschreiben Zusammenhänge zwischen Produkten und Geschmacksstoffen und deren sensorische Wahrnehmungen beim Menschen.

\section{Frühkindliche Geschmacks- prägung}

In den Verhaltenswissenschaften wird davon ausgegangen, dass die ursprüngliche Motivation zur Nahrungsaufnahme (Primärbedürfnisse) bei Neugeborenen durch intensive Körpergefühle (Hunger und Sättigung) gesteuert wird und so eine bedarfsgerechte Versorgung mit Nahrung gewährleistet wird. Was je- doch die Lebensmittelauswahl betrifft, wirken viele unterschiedliche Faktoren auf Kinder (und Erwachsene) ein: Genetische Veranlagung bestimmt die Sensitivität für die Geschmacksrichtungen bitter, süß und umami und auch, was die Empfindlichkeit gegenüber fettigem Geschmack betrifft, gibt es Hinweise für genetische Unterschiede. Diese Sensitivität beeinflusst die Präferenz und Auswahl von Lebensmitteln. Angeborene Präferenzen (z. B. für süßen Geschmack) und Aversionen (z. B. gegen bitteren Geschmack) sind zum Überleben wichtig und hindern uns beispielsweise daran, potenziell schädliche Lebensmittel zu essen. Präferenzen und Aversionen lassen sich aber auch durch Erfahrungen verändern. Dementsprechend spielt nicht nur die Veranlagung, sondern auch die Umwelt bzw. das Umfeld eine Rolle bei der Entwicklung von Präferenzen und Lebensmittelauswahl. So ist das Kind beispielsweise bereits im Mutterleib über das Fruchtwasser - je nach Ernährung der Mutter verschiedenen Geschmacksreizen ausgesetzt. Diese flüchtigen Aromastoffe aus der maternalen Ernährung können auf das Fruchtwasser und postpartal auf die Muttermilch übertragen werden und zu deren Aromatisierung beitragen. Solche Geschmackserfahrungen können sich prägend auf das zukünftige Ernährungsverhalten auswirken (• Abb. 1). Die intrauterine Umgebung hat demnach einen lebenslangen Einfluss auf Geschmackspräferenzen und einen gesunden Ernährungsstil.

Gestillte Babys sind einer wesentlich größeren Bandbreite an Aromastoffen ausgesetzt, als mit Formulanahrung ge- 


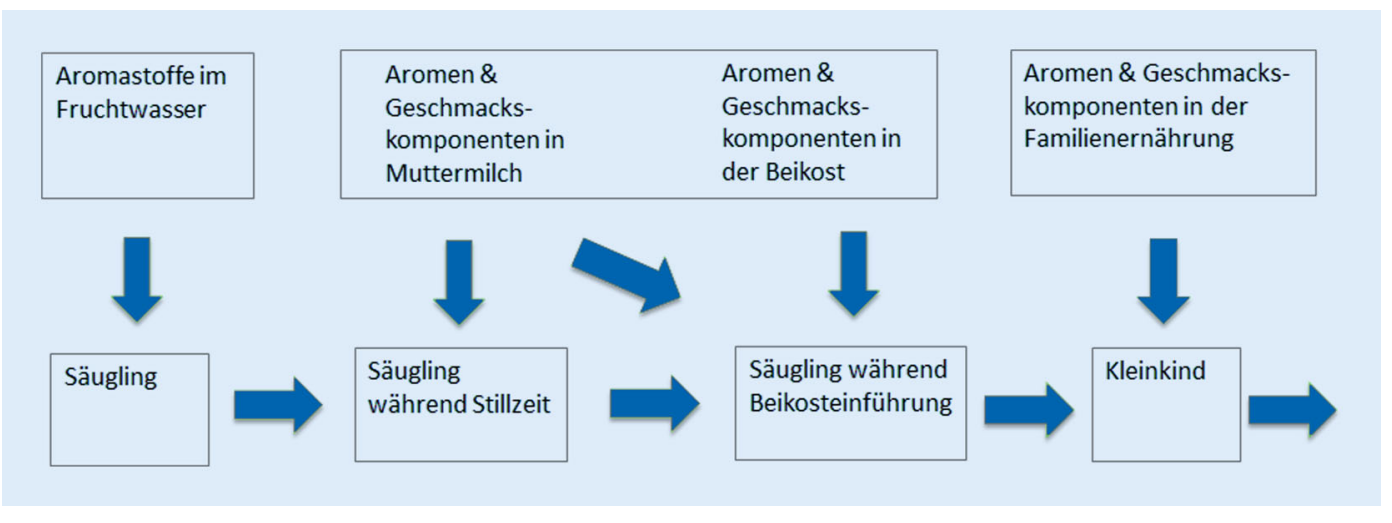

Abb. $1<$ Geschmacksprägende Triggerfaktoren aus der Nahrung im zeitlichen Verlauf von der pränatalen Phase bis in die Kindheit (adaptiert nach [1]) fütterte Babys, da Formulanahrung im Gegensatz zu Muttermilch eine konstant gleichbleibende Geschmacksqualität beinhaltet. Bei ungestillten Kindern kann es daher zu einer möglichen Beeinflussung der Geschmackspräferenzen und weiteren Nahrungsmittelauswahl kommen. Besonders anschaulich wurde dies in einer Studie von Haller et al. präsentiert: Erfahrungen mit Vanillearoma in Formulanahrung im Säuglingsalter führten zu langfristigen Geschmackspräferenzen von Vanille im Erwachsenenalter [2].

\section{Geschmackspräferenzen von Geburt an}

Die Geschmackspräferenz für süßbesteht von Geburt an, da Süße das Vorhandensein von Zuckerarten und verwertbarer Energie indiziert. Die Prädisposition für salzig entwickelt sich erst im Laufe der ersten vier Lebensmonate. Interessanterweise konnte gezeigt werden, dass eine Salzpräferenz bei ausschließlich gestillten Säuglingen nach sechs Monaten besteht, obwohl der Salzgehalt der Muttermilch gering ist. In einer weiteren Studie konnte ein Zusammenhang zwischen Geburtsgewicht des Kindes und seiner Salzpräferenz im Vorschulalter gefunden werden. Crystal und Bernstein zeigten, dass die Salzpräferenz von Säuglingen und der spätere Salzkonsum von Kindern erhöht sind, wenn deren Mütter während der Schwangerschaft unter wiederholtem Erbrechen litten [3, 4]. Auch konnte bei Tierversuchen nachgewiesen werden, dass ein hoher Salzkonsum der Mutter während der Schwangerschaft einen Einfluss auf die Salzpräferenz der Jungen und einen damit verbundenen höheren Salzkonsum hatte. Hingegen zeigen sich Säuglinge gegenüber der sensorischen Präsentation von Fett indifferent - es wird angenommen, dass bei ihnen noch keine Fettpräferenz präsent ist oder sie Fette noch nicht schmecken können.

\section{Übergewicht und Geschmacks- präferenzen der Eltern}

Geschmack ist einer der wichtigsten Faktoren in der Auswahl von Nahrungsmitteln. Obwohl die Entstehung von Adipositas ein multifaktorielles Ereignis ist, gibt es Hinweise darauf, dass bei Übergewichtigen eine Verschiebung von Geschmackswahrnehmung und Geschmackspräferenzen stattfindet und es dadurch unter anderem zur veränderten Nahrungsmittelauswahl kommt und ein Beitrag zur Entstehung der Adipositas geleistet wird. Vor diesem Hintergrund stellt sich nun die Frage, ob und in welchem Umfang eine veränderte Nahrungszusammensetzung in Richtung „western diet“ einen Einfluss auf diese frühen prä- und postnatalen Geschmacksprägungen des Kindes ausübt.

Übergewichtige scheinen eine höhere Affinität zu süßen Lebensmitteln zu zeigen, was einerseits durch Geschmacksunterschiede im Vergleich zu Normalgewichtigen, aber auch durch die Genetik und Umweltfaktoren bedingt sein kann. Übergewichtige und adipöse Personen besitzen im Vergleich zu Normalgewichtigen eine geringere Sensitivität gegenüber süß. AndereStudien jedoch konnten keinen Unterschied in der Geschmacks- wahrnehmung von süßbei Menschen mit hohem BMI im Vergleich zu Normalgewichtigen feststellen. Donaldson et al. und Reed und McDaniel weisen aber darauf hin, dass diese gegensätzlichen Ergebnisse aufgrund unterschiedlicher methodischer Ansätze zustande kommen [5, 6]. Geschlechtsspezifische Unterschiede kommen ebenfalls zum Tragen: Die Süßpräferenz bei erwachsenen Frauen ist höher, als bei erwachsenen Männern. Dieser Unterschied ist bei übergewichtigen Frauen und Männern noch stärker ausgeprägt. Drewnowski et al. und auch Reed und McDaniel weisen darauf hin, dass die Geschmackswahrnehmung von süß sehr variabel sein kann und unter anderem von genetischen Faktoren oder der Anzahl der vorhandenen Geschmacksrezeptoren im Mund abhängt $[6,7]$.

Durch die Geschmackswahrnehmung von süß alleine kann der Zusammenhang zwischen vermehrtem Verzehr süßer Lebensmittel und Übergewicht nicht erklärt werden. Der Fettgehalt eines Lebensmittels muss als zusätzlicher sensorischer Stimulus in Betracht gezogen werden, wobei die sensorische Wahrnehmung von Fett ein Komplex aus subtilen Geschmackswahrnehmungen, Textureigenschaften und Geruchseigenschaften des Lebensmittels ist. Lebensmittel, welche einen hohen Fettgehalt aufweisen, werden als wohlschmeckender wahrgenommen und lieber gemocht, wenn sie zusätzlich gesüßt sind. Ein Effekt, der auch bei Übergewichtigen auftritt. Fett ist zwar nicht als Grundgeschmacksart definiert, es bestehen jedoch starke Hinweise, dass freie Fettsäuren im Mund zum Geschmack eines Lebensmittels beitragen. Studien zeigen, dass Personen mit einer 
geringeren Sensitivität gegenüber Fettsäuren durchschnittlich mehr Fett und fette Lebensmittel zu sich nahmen und einen höheren BMI aufwiesen als die Kontrollgruppe. Auch die Fettpräferenz spielt in der Entwicklung von Adipositas eine Rolle - sie scheint einen größeren Einfluss auf das Körpergewicht zu haben als die Präferenz für Süßes alleine. Studien zeigen, dass ein Zusammenhang zwischen der Präferenz für süß-fette Lebensmittel und Gewichtszunahme besteht.

Der Zusammenhang zwischen Salzpräferenz und Übergewicht ist in der Literatur nur wenig untersucht und die Ergebnisse lassen bis dato noch keine eindeutigen Rückschlüsse zu. Studien konnten aber zeigen, dass adipöse Menschen im Vergleich zu Normalgewichtigen mehr Nahrungsenergie aus Lebensmitteln beziehen, die als "salzig“ gelten, adipöse Jugendliche und Kinder eine reduzierte Salzschwelle besitzen und damit den Schluss nahelegen, dass eine veränderte Salzsensitivität bei Übergewichtigen im Ernährungsverhalten eine Rolle spielen kann.

\section{Pilotstudie des Health Perception Labs}

Seit Jänner 2014 führt eine interdisziplinäre Forschergruppe des Health Perception Labs eine Studie zur Identifikation von frühkindlichen Risikofaktoren für Adipositas durch. Im Rahmen dieser Pilotstudie werden rund 60 gesunde Schwangere und deren Säuglinge von der 24. Schwangerschaftswoche bis zur 16. Lebenswoche des Säuglings begleitet und untersucht. Dabei werden Methoden getestet, die ausgewählte Faktoren der metabolischen Programmierung wie Körperzusammensetzung, Geschmacksentwicklung und Geschmacksprägung in den ersten Lebensmonaten untersuchen. Die angewendeten Methoden umfassen ausführliche medizinische Anamnesegespräche, die Erhebung von Biomarkern aus Blut und Stuhl von Mutter und Säugling bzw. aus der Muttermilch, die potenziell mit Adipositas in Verbindung stehen (u. a. Adipokine, Eicosanoide), Geschmacksschwellen der Mutter für süß und salzig, Geschmackspräferenzen sowie Ernährungsverhalten von Mutter

Paediatr Paedolog 2016 -51:156-161 DOI 10.1007/s00608-016-0396-2

(c) Der/die Autor(en) 2016. Dieser Artikel ist eine Open-Access-Publikation.

\section{Peterseil · W. Gunzer · B. Fuchs-Neuhold \\ Einflussfaktoren auf die Geschmacksentwicklung von Säuglingen}

\section{Zusammenfassung}

Die steigende Prävalenz von Adipositas ist eine der größten Public-Health-Herausforderungen, die Gesundheitssysteme weltweit zu bewältigen haben. Obwohl die Entstehung von Adipositas ein multifaktorieller Prozess ist, gibt es Hinweise darauf, dass bei Übergewichtigen eine Veränderung von Geschmackswahrnehmung und Geschmackspräferenzen stattfindet. Dadurch kann es zu einer veränderten Nahrungsmittelauswahl kommen, die sich auf die Entstehung von Adipositas auswirken kann. Die gesundheitsorientierte Sensorik untersucht den Zusammenhang zwischen Adipositas, Nahrungsmittelauswahl und veränderten Geschmackspräferenzen. Bereits im frühen Kindesalter (prä- und postnatal) entwickeln sich Muster der Geruchs- und Geschmackswahrnehmung. Inwieweit sich eine bestehende maternale Präferenz und/oder veränderte Geschmackswahrnehmung für Lebensmittel mit hohem Zucker- und/oder Fettgehalt auf die Geschmackspräferenz bei Säuglingen und damit auf die spätere Nahrungsmittelauswahl des Kindes auswirkt, ist noch nicht ausreichend geklärt. Im laufenden Pilotprojekt des Health Perception Labs werden daher sensorische Methoden entwickelt, um mögliche Verbindungen zwischen dem Essverhalten der Mutter und den Nahrungsvorlieben der Kinder herstellen zu können.

\section{Schlüsselwörter}

Sensorische Lebensmittelwissenschaft . Gesundheitsorientiert · Perinatale Programmierung $\cdot$ Säuglinge $\cdot$ Adipositas

\section{Factors influencing taste sensation development in infants}

\section{Abstract}

The increasing prevalence of obesity is one of the greatest public health challenges healthcare systems are facing worldwide. Although the development of obesity is a multifactorial process, there is evidence that a shift in taste perception and flavor preferences takes place in obese persons. Such shifts can lead to changes in food selection, which may contribute to the development of obesity. Therefore, healthoriented sensory science is trying to examine the relationship between obesity, food choice and (altered) taste preferences. Patterns of taste and smell perception are shaped early in the prenatal and postnatal development phases. However, the impact of an altered maternal perception or maternal preferences for foods high in sugar and/or fat content on the taste preference in infants has yet to be investigated. Thus, the effect on the subsequent food choices of children also remains unexplored. Therefore, the current pilot project of the Health Perception Lab aims to develop sensory methods in order to find possible associations between the eating behavior of mothers and the food preferences of infants and children.

\section{Keywords}

Food sensory science $\cdot$ Health-oriented . Perinatal programming $\cdot$ Infants · Obesity und Säugling. Schließlich werden auch die Gewichtsentwicklung der Mutter sowie die Messung des Körperfettanteils des Säuglings analysiert. Darüber hinaus werden mimische und gestische Reaktionen des Säuglings auf Geschmacksreize durch audiovisuelle Tools aufgezeichnet und anschließend mittels Facial Action Coding System (FACS) analysiert. Sobald der Säugling das erste Lebensjahr vollendet hat, erfolgt ein Online-Follow-up zum Gesundheitsverhalten der Mutter sowie zum Gesundheitszustand des Säuglings, um längerfristige Daten in die Analysen miteinbeziehen zu können. Erste Studienergebnisse werden im Sommer 2016 erwartet. Mit den aus der Pilotstudie gewonnenen Erkenntnissen sollen Prädiktoren für Adipositas etabliert werden, die Biomarker und anthropometrische Daten berücksichtigen. Ein weiterer Schwerpunkt beinhaltet die Entwicklung von gesunden Lebensmitteln für Kinder und verfolgt einen umfassenden Zugang von Produktdesign über Lebensmittelzusammensetzung, senso- 
Hier steht eine Anzeige.

\section{曾 Springer}


rische Analysen bis hin zur Erhebung der Akzeptanz bei Konsumenten. Zusätzlich werden Lehr- und Lernkonzepte, vor allem für Multiplikatoren im $\mathrm{Ge}$ sundheitsbereich, erstellt. Hierbei sollen wissenschaftlich fundierte Erkenntnisse für Multiplikatoren aufbereitet werden, um so einen niederschwelligen $\mathrm{Zu}$ gang für Adipositasrisikogruppen $\mathrm{zu}$ gewährleisten. Durch diesen Zugang wird im Health Perception Lab angewandte Sensorikforschung mit Fragen der Gesundheitsförderung, Prävention und Therapie kombiniert.

\section{Frühe Geschmacksentwicklung - Prävention}

Bis dato ist nicht geklärt, inwieweit sich eine eventuell bestehende maternale Präferenz und/oder veränderte Geschmackswahrnehmung für fette Lebensmittel mit hohem Zucker- und/oder Fettgehalt auf die pränatale und postnatale Geschmackspräferenz bei Säuglingen und damit auf die spätere Nahrungsmittelauswahl des Kindes auswirkt. Die gesundheitsorientierte Sensorik kann hierzu wichtige neuartige Erkenntnisse liefern. Neben dem sensorischen Einfluss der Eltern spielen diese aber auch durch die Lebensmittelauswahl beim Einkauf eine wesentliche Rolle bei der Verfügbarkeit von Lebensmitteln für ihre Kinder. Die Kinder selbst können dies hauptsächlich durch Ablehnung von Speisen beeinflussen bzw. einschränken.

Unser Konsumverhalten wird durch viele unterschiedliche Faktoren beeinflusst, z. B. Werbung, Peers und Erfahrungen mit Marken. Marketing und Werbung zielen bereits bei Kindern darauf $a b$, die Kaufentscheidungen (der Eltern) zu beeinflussen. Lebensmittelwerbung für Kinder betont vor allem mit den Faktoren "Spaß/Unterhaltung“ und „Geschmack“. Dabei werden fantasievolle Methoden eingesetzt, die die Kinder zum Teil aktiv involvieren, z. B. Spiele, Rätsel, Fantasiefiguren.

Lebensmittel, die speziell für Kinder beworben werden, sind z. B. Zerealien, (süße) Getränke und Snacks. Studien zeigen, dass Lebensmittelwerbung die Präferenzen von Kindern für (Marken-)Lebensmittel beeinflusst, sowohl bei nor- malgewichtigen als auch bei übergewichtigen Kindern. Zwar treffen bei Kindern in der Regel die Eltern Kaufentscheidungen für Lebensmittel, diese werden jedoch stark von ihren Kindern beeinflusst und tendieren dazu, Lebensmittel nach der Akzeptanz ihrer Kinder auszuwählen.

\section{Ernährungsbezogene Interventionen}

Aus diesem Grund ist es begrüßenswert, dass inzwischen ernährungsbezogene Interventionen in Kindergarten und Schule an Bedeutung gewinnen. Nicht nur das Zurverfügungstellen von Obst und Gemüse bzw. Belohnungsansätze fördern Obst- und Gemüseverzehr bei Kindern, sondern auch die Beschäftigung mit sensorischen Aspekten der Lebensmittel. Auf diesem Weg können Kinder ihnen bis dahin unbekannte Lebensmittel auf spielerische Weise kennenlernen. Die Akzeptanz und Neugier auf neue Sorten steigen und damit auch die Wahrscheinlichkeit, Präferenzen für diese Lebensmittel zu entwickeln. So bestehen große Chancen, einerseits einen Grundstein für eine gesundheitsförderliche Ernährungsweise zu legen und andererseits bereits frühzeitig die Entstehung von Übergewicht und Adipositas effektiv einzudämmen.

\section{Fazit für die Praxis}

Empfehlenswert ist es, in der Praxis diese Interventionen möglichst interdisziplinär (ÄrztInnen, DiätologInnen, PädagogInnen, PsychologInnen, Hebammen u. a. m.) zu gestalten und eine möglichst breite Abdeckung zu erreichen (Kooperation mit Krankenkassen, Kindergärten, Hebammenzentren etc.).

\begin{tabular}{l} 
Korrespondenzadresse \\
$\begin{array}{l}\text { B. Fuchs-Neuhold, BSc MSc } \\
\text { Health Perception Lab, } \\
\text { Institut Diätologie, FH } \\
\text { Joanneum } \\
\text { Eggenberger Allee 11, } \\
\text { 8020 Graz, Österreich } \\
\text { bianca.fuchsneuhold@fh- } \\
\text { joanneum.at }\end{array}$ \\
\hline
\end{tabular}

Open access funding provided by FH Joanneum University of Applied Sciences.

\section{Einhaltung ethischer Richtlinien}

Interessenkonflikt. M. Peterseil, W. Gunzer und B. Fuchs-Neuhold geben an, dass kein Interessenkonflikt besteht.

Dieser Beitrag beinhaltet keine von den Autoren durchgeführten Studien an Menschen oder Tieren.

Open Access Dieser Artikel wird unter der Creative Commons Namensnennung 4.0 International Lizenz (http://creativecommons.org/licenses/by/4.0/deed de) veröffentlicht, welche die Nutzung, Vervielfältigung, Bearbeitung, Verbreitung und Wiedergabe in jeglichem Medium und Format erlaubt, sofern Sie den/die ursprünglichen Autor(en) und die Quelle ordnungsgemäßnennen, einen Linkzur Creative Commons Lizenz beifügen und angeben, ob Änderungen vorgenommen wurden.

\section{Literatur}

1. Beauchamp GK, Menella JA (2009) Early flavor learning and its impact on later feeding behavior. J Pediatr Gastroenterol Nutr 48:25-30

2. Haller R, Rummel C, Henneberg S, Pollmer U, Koster EP (1999) The influence of early experience with vanillin on food preference later in life. Chem Senses 24(4):465-467

3. Crystal SR, Bernstein IL (1995) Morning sickness: impact on offspring salt preference. Appetite 25(3):231-40

4. Crystal SR, Bernstein IL (1998) Infant salt preference and mother's morning sickness. Appetite 30(3):297-307

5. Donaldson LF, Bennett L, Baic S, Melichar JK (2009) Taste and weight: is there a link? Am J Clin Nutr 90(3):800S-803S

6. Reed DR, McDaniel AH (2006) The human sweet tooth. BMC Oral Health doi:10.1186/1472-6831-6S1-S17

7. Drewnowski A, Mennella JA, Johnson SL, Bellisle F (2012) Sweetness and food preference. J Nutr 142(6):1142-1148 
Hier steht eine Anzeige.

\section{曾 Springer}

